\title{
Backward diode composed of a metallic and semiconducting nanotube
}

\author{
Ryo Tamura \\ Department of Physics, Graduate School of Science, University of Tokyo, Hongo 7-3-1, Bunkyo-ku, Tokyo 113, Japan
}

The conditions necessary for a nanotube junction connecting a metallic and semiconducting nanotube to rectify the current are theoretically investigated. A tight binding model is used for the analysis, which includes the Hartree-Fock approximation and the Green's function method. It is found that the junction has a behavior similar to the backward diode if the gate electrode is located nearby and the Fermi level of the semiconducting tube is near the gap. Such a junction would be advantageous since the required length for the rectification could be reduced. 
Carbon nanotubes (NTs) 1 are promising components for nano-electronics because they can either be semiconducting $(\mathrm{S})$ or metallic $(\mathrm{M})^{2}$. This has been explored in various experiments, for example, NT transistors $\mathrm{B}$, NT diodes 4 , and NT junctions 5 . The NT junctions made by a pentagon-heptagon defect pair can be classified into three types: MM, MS, and SS: M and S stand for a metallic NT and a semiconducting NT, respectively. It is remarkable that all the chemical bonds of NT junctionsare essentially $s p^{2}$ orbitals of the carbon with no impurity 4 , which favors a coherent electron flow through the junction. For MM-NT junctions, a scaling law of the electronic transport was found 1 . On the other hand, MSNT junctions are expected to cause the Schottky barrier which is important to rectify the current 10 . In this work, we focus on MS-NT junctions connecting the $(9,0)$ tube and the $(8,0)$ tube shown in Fig.1. Though in real experiments, these junctions are sometimes bent 6 , we investigate straight junctions because the difference does not alter the results qualitatively as was explained in Ref.10.

For NT devices, the gate electrode is quite important for controlling the transport properties, as was shown in an experiment where an AFM tip was used as the gate electrode 1 . It was recently pointed out that the length of the NT junction needs to be larger than the nanoscale in order to rectify the current becaupepf the large depletion width in the underdoped case 10 12. An important prospect is to understand whether or not the depletion width can be made narrower by approaching the gate closer to the NT junction. In this paper, we demonstrate that this could indeed be possible because the gate electrode screens the Coulomb interaction in the junction. The main purpose of this paper is to support the validity of this method.

The current flowing through MS-NT junctions can be quite sensitive to the details of the Schottky barrier caused by the Coulomb interaction. Though MS-NT junctions have been recently investigated theoretically 10.13 the Coulomb interaction was not considered in Ref.13 and the semi-classical model used in Ref.L is not appropriate when the spatial range of the potential variation is short. For these reasons we have adopted a tight binding model with $\pi$ orbitals and the Hartree-Fock approximation 14 . The corresponding Hamiltonian $H$ is represented with the density matrix $\rho_{i, j}$ by

$H_{i, j}=t_{i, j}-\frac{1}{2} U\left(\left|\vec{r}_{i}-\vec{r}_{j}\right|\right) \rho_{i, j}+\delta_{i, j} \sum_{k} U\left(\left|\vec{r}_{i}-\vec{r}_{k}\right|\right)\left(\rho_{k, k}-1\right)$

where $\vec{r}_{j}$ is the position of the carbon atom $j$ which can be determined approximately by the condition that all bonds must essentially have the same length. This is justified since the detailed atomic structure do not modify significantly the electronic structure 8 . The first term in Eq. (1) has a nonzero constant value $-t<0$ when $i$ and $j$ are nearest neighbors and is zero otherwise. The second and third term are the exchange and Hartree term, respectively. The Coulomb interaction $U(r)$ is assumed to be $U(r)=t / \sqrt{1+4(r / a)^{2}}$ where $a$ is the bond length.

The Green's functipn method is used for calculating the transmission rate 15.16 . The system is divided into three regions: the region near the defects $(C)$, the $(8,0)$ tube far from the defects $(S)$, and the $(9,0)$ tube far from the defects $(M)$. The corresponding Hamiltonian $H$ is written as $H=H_{C}+H_{S}+H_{M}+\tilde{t}_{S}+\tilde{t}_{M}$, where $\tilde{t}_{p}$ $(p=S$ or $M)$ represents the off-diagonal elements between the $C$ region and the $p$ region. For each region $p$, the matrix element $\left(H_{p}+\tilde{t}_{p}\right)_{i, j}$ is written as $\tau_{p}$ when $i$ and $j$ are nearest neighbors, $\epsilon_{p}$ when $i=j$, and zero otherwise. For each iteration step, the parameter $\epsilon_{p}$ and $\tau_{p}$ are determined by the condition that the near and far region are connected smoothly, i.e. $\epsilon_{p}$ and $\tau_{p}$ are taken to be the same as the corresponding averaged value of $\left(H_{C}\right)_{i, j}$ with the atom $j$ directly connected to the region $p$. The retarded Green's function $G(E)=(E+i \delta-H)^{-1}$ can then be represented by

$$
G_{k, l}=\left(E-H_{C}-\Lambda_{S}-\Lambda_{M}\right)_{k, l}^{-1},
$$

where $k$ and $l$ belong to the region $C$. The effect of the region $S$ and $M$ are included in the 'self energies', $\Lambda_{p} \equiv \tilde{t}_{p}\left(E+i \delta-H_{p}\right)^{-1} \tilde{t}_{p}$. From Eq.(2), the density matrix $\rho$ in the region $C$ can be calculated as

$$
\rho=\frac{1}{\pi} \int_{E_{F 1}}^{E_{F 2}} G \Gamma_{2} G^{*} d E+\frac{1}{\pi} \int_{-\infty}^{E_{F 1}} G\left(\Gamma_{2}+\Gamma_{1}\right) G^{*} d E .
$$

Here $\Gamma_{p} \equiv i\left(\Lambda_{p}-\Lambda_{p}^{*}\right), E_{F 1} \equiv \min \left(E_{F M}, E_{F S}\right)$ and $E_{F 2} \equiv$ $\max \left(E_{F M}, E_{F S}\right)$. Thus, $H$ and $\rho$ can be calculated selfconsistently with Eqs.(11), (2), and (3). It is worth noting that the integrand of the second term in $\mathrm{Eq}\left(\mathrm{P}_{0}\right)$ equals the DOS because $G\left(\Gamma_{S}+\Gamma_{M}\right) G^{*}=i\left(G-G^{*}\right)$.

Although the electron density $\rho_{i, i}$ in Eq.(3) is only defined for the region $C$, the Hartree terms caused by the charges outside this region should be included in $H_{C}$. This can be done by repeating periodically that NT unit cell in the region $C$ which is nearest to the far region. Furthermore the electrostatic potential caused by the gate electrode can be obtained by the image charge method. Here we can choose the electrostatic potential to be zero at the gate surface without loss of generality because the difference, not the absolute value, of the electrostatic potential is relevant quantity. Note that the nanotubes are not neutral by themselves, but the total system including the image charge is neutral. In our model, the gate is planar and parallel to the tube axis. Its distance from the tube axis is chosen to be three times the radius of the $(9,0)$ tube This is much smaller than the gate separation in Ref 10 . At this distance, we have verified that the orientation of the defects does not change our main results. In the following, the defects are assumed to face the gate (see Fig.11). 
When self-consistency has been achieved, the current $I$ is calculated by

$$
I=(2 e / h) \int_{E_{F M}}^{E_{F S}} \operatorname{Tr}\left[\Gamma_{S} G \Gamma_{M} G^{*}\right] d E .
$$

The integrand of Eq. (14) equals the total transmission rate $T(E) \equiv \sum_{i, j} T_{i, j}(E)$ where $T_{i, j}$ is the transmission rate from the $i$ th channel to the $j$ th channel. In the energy range considered here, both tubes have two channels except for the gap region of the $(8,0)$ tube. Note that $T(E)$ may be larger than one, though $T_{i, j} \leq 1$.

The electron potential at each atom site is shown by circles in Fig.2. The Fermi levels $\left(E_{F M}, E_{F S}\right)$ shown by straight

broken lines are $(-0.230 t,-0.344 t),(-0.480 t,-0.344 t)$, $(-0.370 t,-0.480 t)$ and $(-0.600 t,-0.480 t)$ in (a), (b), (c) and (d), respectively. These four situations are referred to(a),(b),(c) and (d), hereafter. The horizontal axis is the position along the tube axis and its range is as same as the length of the region $C$. In Fig.2, the local gap energy region (hatched area) and the valence band edge (thin curved broken line) in the $(8,0)$ tube are determined by the averaged electron potential and the gap width of $H_{S}$. The Fermi levels correspond to hole doping which is not due to dopant atoms, but caused by the source, drain and gate electrodes. The regions occupied by the electrons are shown by gray area. Though the energy range between $E_{F S}$ and $E_{F M}$ is partially occupied (this corresponds to the first term of Eq.(3)), it is not shown in Fig.2 explicitly. One may notice that several circles show a sharp increase or rapid drop near the interface in Fig.2. These abrupt changes are caused by an excess of the electron on the pentagon and a deficiency on the heptagonitiv.

The origin of the band bending seen in Fig.2 (b) and (d) is explained qualitatively with the 'shifted Fermi level', $\tilde{E}_{F p} \equiv E_{F p}-\epsilon_{p}$, as follows. Using the energy integral of the DOS, the hole density in the far regions can be approximated by $\rho_{M}^{h} \sim C_{M}\left|\tilde{E}_{F M}\right|$ and $\rho_{S}^{h} \sim C_{S} \sqrt{\tilde{E}_{F S}^{2}-\Delta^{2}}$ with the half gap width $\Delta$. Here $C_{M} \simeq C_{S}$ because the two tubes have similar radii. Then approximate values of the ratio $\rho_{S}^{h} / \rho_{M}^{h}$ are 0.32 and 0.44 for Fig.2(b) and (d), respectively, so $\rho_{S}^{h}<\rho_{M}^{h}$ in the both situations. The charge distribution connecting $\rho_{M}^{h}$ to $\rho_{S}^{h}$ causes the electron potential to decrease toward the metallic tube, and is responsible for the band bending. Comparing Fig.2(b) and (d), one can see that both the height and the width of the barrier decrease as the hole density $\rho_{S}^{h}$ increases. Qualitatively, this relation is similar to that found in Ref.10.

The inset of Fig. 3 shows the dependence of the current $I$ on the bias voltage $V_{s d} \equiv\left(E_{F M}-E_{F S}\right) /|e|$. The six open triangles and the five closed circles represent the data when $E_{F S}=-0.344 t$ and $E_{F S}=-0.480 t$, respectively. The data corresponding to Fig.2 2 are indicated by the arrows. The horizontal separation of the triangles or the circles is not regular because each Fermi level $E_{F p}$ was calculated with a fixed shifted Fermi level $\tilde{E}_{F p}$. This was done since the constant $\tilde{E}_{F p}$ keeps the $\rho_{p}^{h}$ unchanged in the iteration so that it is advantageous to achieve selfconsistency.

The open triangles in the inset of Fig. 3 indicate that the current with constant $E_{F S}=-0.344 t$ is rectified, i.e. the resistance is much higher when $V_{s d}<0$ than when $V_{s d}>0$. For example, $V_{s d} / I \sim 32 \mathrm{k} \Omega$ for the case (b) and $V_{s d} / I \sim 1300 \mathrm{k} \Omega$ for the case (a). A positive $V_{s d}$, however, corresponds to a reverse bias for the thermoelectron current in conventional MS junctions, i.e. the barrier becomes higher as $V_{s d}$ increases as can be seen in Fig. 2. The mechanism of this reversed rectification is similar to that of the backward diode 18 , which can be understood by examining the total transmission rate $T(E)$ as a function of the energy (Fig. 3). Since the necessary energy range to calculate $I$ with Eq.(1) is between $E_{F M}$ and $E_{F S}$, lines in Fig. 3 are limited to this range. $T(E)$ in the case (b) is considerably larger for the whole bias window because the spatial thickness of the barrier is reduced due to the proximity of the gate (see Fig.2 (b)). This leads to a large current for positive $V_{s d}$. If the barrier had been much thicker as in Ref.10, the current would have been close to zero. On the other hand, $T(E)$ in the case (a) becomes zero for most of the bias window since the energy gap of the $(8,0)$ tube blocks the current as can be seen in Fig 2 (a). This example makes it clear that the necessary condition for keeping the current small when $V_{s d}$ is negative is to fix $E_{F S}$ near the energy gap. Since $E_{F S}=-0.344 t$ is closer to the energy gap than $E_{F S}=-0.480 t$, the triangles show much smaller current than the circles in the inset when $V_{s d}<0$. This means that a better rectification has been achieved in the former case. Here we should mention that $-\left(E_{F S}+E_{F M}\right) / 2$ was fixed, i.e. $E_{F S}$ depends on $V_{s d}$, in each I-V curve in Ref.E. This is not suitable for the rectification presented here.

In the present paper, we have to use a relatively smaller Coulomb interaction $U(r)=t / \sqrt{1+4(r / a)^{2}}$ than that estimated in other papers 44 , since the long spatial range of $U(r)$ makes the convergence difficult. $U(r)$ is probably larger in the actual experiments than that used here, but it is expected that values of larger $U(r)$ favor our conclusion. In fact, it is well known that the screening length $W$ is determined by the relation $W \propto 1 / \sqrt{U(0) N_{F}}$ where $N_{F}$ is the DOS at the Fermi level. Though this relation itself might not hold for MS-NT junctions, a decrease of $W$ with a simultaneous increase of $U(0)$ will hold and favor our conclusion.

In summary, we have calculated the I-V characteristics of a typical MS-NT junction. It is found that approaching the gate electrode to the junction and fixing $E_{F S}$ near the energy gap could be an effective method to overcome the problem of the large depletion length 1 , 12 . The rectifying MS-NT junction discussed here is analogous to the backward diode. Though density functional calculations 
will ultimately be necessary, the results obtained here are worth testing in experiments.

The author wishes to thank Professor Masaru Tsukada for stimulating discussions. He also acknowledges Michel Gauthier for reading the manuscript and Shousuke Nakanishi for his technical support on the computer. This work was supported in part by the Grant-in-Aid for Scientific Research on Priority Area "Fullerenes and Nanotubes" by the Ministry of Education, Science and Culture of Japan.

FIG. 1. The junction connecting the $(9,0)$ tube and the $(8,0)$ tube. Above right: setup of the electronic circuit.

FIG. 2. The electron potential energy at each atom site. The Fermi levels $\left(E_{F M}, E_{F S}\right)$ shown by straight broken lines are $(-0.230 t,-0.344 t), \quad(-0.480 t,-0.344 t)$, $(-0.370 t,-0.480 t)$ and $(-0.600 t,-0.480 t)$ in (a), (b), (c) and (d), respectively. The unit of the vertical axis is the transfer integral $t$. The horizontal axis represents the position along the tube axis and its unit is the lattice constant of graphite, i.e. $\sqrt{3}$ times the bond length $a$. Its origin is the interface between the $(9,0)$ tube and the $(8,0)$ tube and its range is the same as that of the near region $C$. The horizontal arrows indicate the direction of the electron's flow. The local gap energy region, the valence band edge, and the region occupied by electrons are shown by the thin curved broken line, the hatched area and the gray area, respectively.

FIG. 3. Total transmission rate as a function of energy in the bias window. The unit of the horizontal axis is the transfer integral $t$. Inset: $\mathrm{I}-\mathrm{V}$ characteristics of the junction when $E_{F S}$ is fixed to $-0.344 t$ (open triangles) and $-0.480 t$ (closed circles). The units of the horizontal and vertical axis are $2 e t / h \sim 0.21 \mathrm{~mA}$ and $t /|e| \sim 2.7 \mathrm{~V}$, where $e$ and $h$ are the electron charge and Planck's constant, respectively. Data corresponding to Fig. 2 are indicated by the arrows.

\footnotetext{
${ }^{1}$ S. Iijima, Nature 354, 56 (1991).

${ }^{2}$ N. Hamada, S. Sawada, and A. Oshiyama, Phys. Rev. Lett. 68, 1579 (1992); J. W. Mintmire, B. I. Dunlap, and C. T. White, ibid. 68, 631 (1992); R. Saito, M. Fujita, G. Dresselhaus, and M. S. Dresselhaus, Phys. Rev. B 46, 1804 (1992).

${ }^{3}$ S. J. Tans, A. R. M. Verschueren, and C. Dekker, Nature 393, 49 (1998).

${ }^{4}$ R. D. Antonov and A. T. Johnson, Phys. Rev. Lett. 83, 3274 (1999).

${ }^{5}$ S. Iijima, T. Ichihashi, and Y. Ando, Nature 356, 776 (1992).

${ }^{6}$ Z. Yao, H. W. CH. Postma, L. Balents, and C. Dekker,
}

Nature 402, 273 (1999).

${ }^{7}$ R. Saito, G Dresselhaus, and M. S. Dresselhaus, Phys. Rev. B 53, 2044 (1996); L. Chico, L. X. Benedict, S. G. Louie, and M. L. Cohen, ibid. 54, 2600 (1996); B. I. Dunlap, ibid. 46, 1933 (1992); J. C. Charlier, T. W. Ebbesen, and Ph. Lambin, ibid. 53, 11108 (1996); L. Chico, Vincent H. Crespi, Lorin X. Benedict, Steven G. Louie, and Marvin L. Cohen, Phys. Rev. Lett. 76, 971 (1996).

${ }^{8}$ V. Meunier, L. Henrard, and Ph. Lambin, Phys. Rev. B 57, 2586 (1998).

${ }^{9}$ R. Tamura and M. Tsukada, Phys. Rev. B 55, 4991 (1997); 58, 8120 (1998);61, 8548 (2000); H. Matsumura and T. Ando, J. Phys. Soc. Jpn. 67, 3542 (1998).

10 A. A. Odintsov, Phys. Rev. Lett. 85, 150 (2000).

${ }^{11}$ S. J. Tans and C. Dekker, Nature 404, 834 (2000); A. Bachtold, M. S. Fuhrer, S. Plyasunov, M. Forero, E. H. Anderson, A. Zettl, and P. L. McEuen, Phys. Rev. Lett. 84, 6082 (2000).

${ }^{12}$ F. Leonard and J. Tersoff, Phys. Rev. Lett. 83, 5174 (1999).

13 M. S. Ferreira, T. G. Dargam, R. B. Muniz, and A. Latge, Phys. Rev. B 62, 16040 (2000).

${ }^{14}$ K. Harigaya and S. Abe, Phys. Rev. B 49, 16746 (1994): J. Ma and R. Yuan, ibid. 57, 9343 (1998); A.A.Farajian, K. Esfarjani, and Y. Kawazoe, Phys. Rev. Lett. 82, 5084 (1999).

15 Electronic Transport in Mesoscopic Systems, edited by S. Datta (Cambridge University Press, Cambridge, 1995).

16 S. Sanvito, Y-K. Kwon, D. Tomanek, and C. J. Lambert, Phys. Rev. Lett. 84, 1974 (2000); H.Mehrez, J. Taylor, H. Guo, J. Wang, and C. Roland, ibid. 84, 2682 (2000); D. Orlikowski, M. B. Nardelli, J. Bernholc, and C. Roland, Phys. Rev. B 61, 14194 (2000); M. P. Anantram, S. Datta, and Y. Xue, ibid. 61, 14219 (2000).

${ }^{17}$ R. Tamura, K. Akagi, M. Tsukada, S. Itoh, and S. Ihara, Phys. Rev. B 56, 1404 (1997).

18 Tunneling and Negative Resistance Phenomena in Semiconductors, edited by D. K. Roy (Pergamon Press, New York, 1977). 
This figure "fig1.gif" is available in "gif" format from: http://arxiv.org/ps/cond-mat/0106040v1 
This figure "fig2.gif" is available in "gif" format from: http://arxiv.org/ps/cond-mat/0106040v1 
This figure "fig3.gif" is available in "gif" format from: http://arxiv.org/ps/cond-mat/0106040v1 